\title{
Estrategia de optimización para la síntesis dimensional de un robot paralelo 5R para una aplicación de mesa de corte
}

\section{An approach for optimal dimensional synthesis of a $5 \mathrm{R}$ parallel robot for a CNC X-Y cutter}

\author{
Antonio Ramirez-Matheus ${ }^{1}$, Miguel Díaz-Rodríguez ${ }^{2}$, Octavio Andrés González-Estrada ${ }^{3}$ \\ ${ }^{1}$ Laboratorio de Mecatrónica y Robótica, Departamento de Tecnología y Diseño, Universidad de los Andes, \\ Mérida, Venezuela. Email: antonio238245@gmail.com \\ ${ }^{2}$ Laboratorio de Mecatrónica y Robótica, Departamento de Tecnología y Diseño, Universidad de los Andes, \\ Mérida, Venezuela. Orcid: 0000-0001-7243-4957, Email: dmiguel@ula.ve. \\ ${ }^{3}$ Grupo de Investigación en Energía y Medio Ambiente - GIEMA, Escuela de Ingeniería Mecánica, Universidad Industrial de \\ Santander, Ciudad Universitaria, Bucaramanga, Colombia. Orcid: 0000-0002-2778-3389, Email: agonzale@uis.edu.co
}

RECIBIDO: Abril 15, 2017. ACEPTADO: Mayo 20, 2017. Versión FinAL: Mayo 17, 2017.

\begin{abstract}
RESUMEN
Tareas industriales como el corte $\mathrm{X}-\mathrm{Y}$ pueden ser realizadas por robots planos. En este sentido, el robot paralelo plano de 2 Grados de Libertad (GdL) y 5R puede ser empleado como concepto de diseño. Un robot paralelo está conformado por varias cadenas cinemáticas cerradas, lo que le da una estructura que permite una mejor distribución de las cargas soportadas. Los robots paralelos presumen características ventajosas en cuanto a la relación peso del robot y peso de la carga soportada, tienen mayor velocidad, rigidez y precisión. Como principal contrapartida, los robots paralelos tienen un espacio de trabajo reducido. En este trabajo, se busca diseñar un robot plano de $2 \mathrm{GdL} 5 \mathrm{R}$ de forma tal que su espacio de trabajo sea el máximo posible y que además presente óptima destreza. Para ello, se toma ventaja de la técnica de optimización Algoritmos Genéticos (AG) y se desarrolla un procedimiento numérico para optimizar el espacio de trabajo y la agilidad con que el robot puede moverse (destreza). Dos casos de estudio ejemplifican la implementación del procedimiento propuesto.
\end{abstract}

PALABRAS CLAVE: Robot Paralelo, Algoritmo Genético, Optimización, Destreza.

\begin{abstract}
Planar robots can perform industrial tasks such as CNC X-Y cutter. Among them, the 5R two-degrees-of-freedom (2DoF) planar parallel robot can become a useful conceptual design. Parallel robots are made of several closed kinematic chains, which gives them a structure enabling to bear more payload. These robots present the advantages of better load/size capacity, speed, rigidity and precision, while their main drawback is the limited workspace. The paper aim to design the 5R-2DoF parallel robot so that its reachable workspace will be maximum and also with optimal dexterity. In this sense, we take advantage of the Genetic Algorithms (GA) in order to develop an approach for optimal design of the robot. The paper shows a path to follow to design the robot taking into account a maximum workspace with dexterity. We present two study cases to test the performance of the proposed approach.
\end{abstract}

KEYWORDS: Parallel Robot, Genetic Algorithm, Optimization, Dexterity.

\section{INTRODUCCIÓN}

Dentro de los tipos de robot industriales se encuentran los robots paralelos (RP), que consisten en dos plataformas, una fija y una móvil, conectadas por al menos dos cadenas cinemáticas abiertas que le proporcionan movimiento relativo entre la plataforma móvil y la base. Las cadenas cinemáticas o piernas le proporcionan una mayor rigidez al robot, a expensas de limitar su espacio de trabajo. Haciendo un símil con el cuerpo humano, una

Este artículo puede compartirse bajo la licencia CC BY-ND 4.0 y se referencia usando el siguiente formato: A. Ramirez-Matheus, M. DíazRodríguez, O. A. González-Estrada, "Estrategia de optimización para la síntesis dimensional de un robot paralelo 5R para una aplicación de mesa de corte", UIS Ingenierías, vol. 16, no. 2, pp. 197-206, Julio-Diciembre 2017. Doi: https://doi.org/10.18273/revuin.v16n2-2017018 
cadena cinemática abierta (robot serial) puede ser vista como un solo brazo. El brazo puede realizar diversas tareas; sin embargo, cuando se requiere de mayor fuerza o de precisión el ser humano emplea los dos brazos. Cuando se utilizan los dos brazos se está en presencia de un robot paralelo. Así, la base es el torso y la plataforma móvil equivale al elemento sujetado con ambas manos. Siguiendo este símil, se puede observar que el RP gana mayor capacidad de carga, mayor precisión a expensas de perder capacidad de alcance en el movimiento de los brazos (espacio de trabajo o workspace). Sin embargo, las ventajas que presenta (mayor rigidez y precisión) han convertido los RP en una alternativa indispensable para el diseño de robots tanto en el mundo académico como en la industria [1].

Una de las aplicaciones potenciales de los RP es su empleo como robot de posicionado $x-y$ para aplicaciones de mecanizado, específicamente como sistema de posicionamiento de la herramienta en una mesa de corte. El diseño de una mesa de corte basado en RP motiva el desarrollo de este trabajo. El objetivo del artículo es presentar una estrategia de diseño óptimo para un RP que permita abarcar el mayor espacio de trabajo y que además presente un buen desempeño cinemático en el plano $x-y$. En este sentido, se propone utilizar un RP de 2 GdL de configuración 5R, como mecanismo para posicionamiento de mesa de trabajo $x-y$, donde $\mathrm{R}$ indica que el mecanismo va unido con pares de revoluta $(\mathrm{R})$. Para la optimización se tendrá como criterio el índice local de destreza, por lo que las dimensiones del robot se determinaran a partir de maximizar el área alcanzable y área diestra. El área diestra es aquella que presenta cierta destreza respecto a un índice cinemático que se define más adelante. Para resolver la tarea, se va a tomar ventaja de técnicas de optimización basadas en algoritmos evolutivos (Algoritmos Genéticos, AG), ya que estas técnicas permiten localizar máximos o mínimos globales con lo cual se evita conseguir soluciones optimas locales [2]. Además, puede permitir desarrollar algoritmos para planificación de trayectorias del robot [3]. El AG tiene una serie de parámetros para su implementación, lo que hace necesario realizar un estudio exhaustivo de los operadores y parámetros del AG. Hasta donde los autores de este trabajo han investigado, no se han presentado estudios exhaustivos para el diseño de RP, como el realizado en este trabajo. Por lo que la contribución de este artículo es mostrar una metodología para implementar AG en el diseño de RP. Además, se plantean y detallan dos técnicas (basadas en técnica de Montecarlo y Nube de Puntos) para estimar el área alcanzable y el área de diestra del RP.

Antes de continuar con el desarrollo del trabajo se deben mencionar algunas investigaciones previas importantes.

\section{ANTECEDENTES}

Merlet [4] presenta un método para el diseño de un RP de tipo plataforma Gough el cual es un robot que se utiliza muy a menudo como simulador de vuelo. Si bien el robot Gough presenta 6 grados de libertad (GdL), y en una tarea de posicionamiento el plano solo se requiere $2 \mathrm{GdL}$, los aspectos metodológicos utilizados en [4] permiten servir de orientación para desarrollar la metodología propuesta en este artículo. En la metodología de diseño proponen: 1) las especificaciones de diseño incluyen como requisito el espacio de trabajo 2) se determinan todas las posibles geometría del robot, y 3 ) de entre todas estas geometría se realiza un análisis numérico con el objeto de determinar el robot que cumple de forma adecuada con el espacio de trabajo requerido. Sin embargo, el diseño de un RP no puede limitarse solo al espacio de trabajo, sino que también debe considerar otros aspectos tales como el Jacobiano, la manipulabilidad, el número de condición y la precisión [4].

Los aspectos indicados en [4] y [5] fueron evaluados para el caso específico del RP 5R en [6], donde se advierte que el diseño no puede basarse solo en el análisis del espacio de trabajo, ya que el robot presenta singularidades en el interior de dicho espacio. El trabajo describe los problemas involucrados en el diseño óptimo del mecanismo 5R para realizar su síntesis dimensional, donde se concluye que, para el diseño óptimo del 5R, se debe plantear una optimización multicriterio que incluya espacio de trabajo, singularidades, destreza e índice de acondicionamiento definidos para el caso particular del 5R. Entre los aspectos a destacar, se recomienda considerar que la estructura del robot $5 \mathrm{R}$ sea simétrica con el fin de reducir el número de parámetros a optimizar. Dado a que la síntesis dimensional requiere de un proceso de optimización, los Algoritmos Genéticos (AG) pueden resultar de mucha utilidad ya que permiten obtener el máximo o mínimo global. Los AG ya han sido empleados para la síntesis dimensional de un robot tipo Delta para un espacio de trabajo prescrito [7], y para el diseño de RP de 2GdL PRRRP, donde $\mathrm{P}$ y $\mathrm{R}$ definen el tipo de articulación o junta cinemática. Dicho robot presenta actuadores prismáticos (P) y juntas de revoluta (R) [8]. En la última referencia se describe una metodología del diseño cinemático basándose en criterios de evaluación que implica rigidez, destreza y lo que los autores definen como índice de acondicionamiento global.

Por otra parte, otro de los procedimientos propuestos para el diseño de RP es la optimización basada en el Espacio de Trabajo Efectivo [9], donde se propone maximizar el espacio de trabajo junto con los índices tales como manipulabilidad y destreza. En la referencia [9] lo índices de desempeño cinemático se incluyen en el proceso de 
optimización mediante restricciones, y se emplean técnicas de búsqueda directa para la optimización. También se han desarrollado técnicas multiobjetivo basadas en AG donde se incluye un enfoque orientado a la aplicación (EOA) [10]. En este sentido, en la referencia [11] se presentó una estrategia que emplea AG en el diseño óptimo EOA sobre la base de una aplicación educativa.

Como se aprecia en los antecedentes presentados, el punto fundamental para el diseño de RP viene dado por el indicador utilizado para evaluar su desempeño cinemático. Por lo que en [12] analizan los indicadores y evalúan los distintos conceptos de indicadores cinemáticos usados hasta el momento, las diversas alternativas de cálculo, y las características, virtudes o defectos atribuidos a los mismos. El estudio concluye que el empleo combinado del número de condicionamiento y una medida de la manipulabilidad es común en trabajos sobre diseño óptimo. Un procedimiento para incluir varios índices en el proceso de optimización de consecución de objetivos o goal attainment method. Un ejemplo del procedimiento para el diseño de trayectorias puede ser consultado en [13]. Dichos criterios son considerados como la representación más adecuada de la destreza del robot [14], donde para el diseño de un robot de 2 GdL 5R utilizan el método de búsqueda directo como algoritmo de optimización.

Otro de los aspectos que han sido introducidos en la optimización dimensional de RP es el consumo de energía, lo cual es de interés actual dado que las tecnologías verdes crecen continuamente. En [15] utiliza describe el desarrollo de AG como algoritmo de optimización para obtener un robot de mínimo consumo. Aunque en el presente trabajo no es de interés minimizar el consumo de energía, dicho trabajo es un antecedente de cómo se desarrolla un AG en problemas de optimización de RP. Por último, se debe mencionar la referencia [16] donde han incluido el rendimiento Cinético-Estático como criterio de optimización y empleado el entorno numérico Matlab para el desarrollo de la optimización. Muchos de los trabajos desarrollados emplean el entorno de programación de Matlab, por lo está trabajo se emplea dicho programa para desarrollar los códigos necesarios para ejecutar el algoritmo de optimización propuesto.

\section{METODOLOGÍA}

\subsection{Descripción del robot 2-GdL 5R}

La Figura 1 presenta el diagrama cinemático, así como los parámetros geométricos relevantes para el diseño cinemático del RP propuesto como mesa de corte X-Y. El robot presenta cinco (5) eslabones unidos a través de pares de revolución (R). Tal como se aprecia en la figura, la plataforma base viene definida por la distancia entre los puntos $A C$ con magnitud $d$, la distancia que define los eslabones más cercanos a la base, puntos $A B$ y $C D$, viene dada por $l 1$, y los eslabones más distantes respecto a la plataforma fija, puntos $B P$ y $D P$, viene definido por $l 2$. En este robot, la plataforma móvil se limita al punto $p$. Se emplean coordenadas cartesianas $(x, y)$ para localizar el punto $p$ y coordenadas de junta $(\theta 1, \theta 2, \theta 3, \theta 4)$ para las cadenas cinemáticas abiertas.

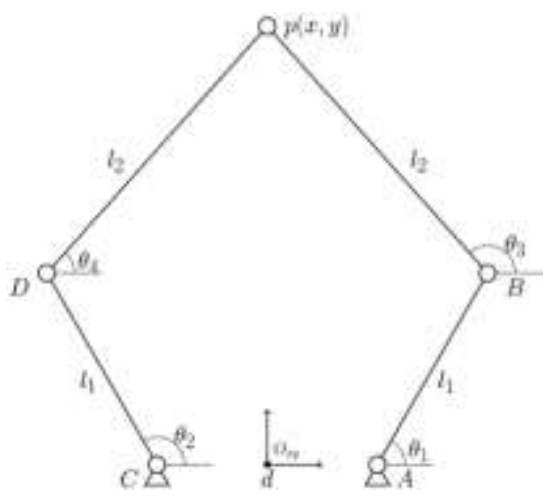

Figura 1. Diagrama Cinemático del Robot Paralelo de 2 GdL 5R.

Tal como se propone en [12] y [14] la optimización del RP 5R considerará como objetivo lograr un máximo espacio de trabajo y a su vez una buena destreza. Para construir la función objetivo es necesario desarrollar una estrategia para estimar tanto el espacio de trabajo como la destreza. Para ello primero se deben desarrollar las ecuaciones de la cinemática inversa y el Jacobiano del robot [17]. La cinemática inversa es la base para estimar el espacio de trabajo mediante la estrategia propuesta en este artículo, y el número de condición del Jacobiano del robot será la medida empleada para la destreza.

\subsection{Problema de posición inverso y Jacobiano del robot 2-GdL 5R}

Se parte de la hipótesis de que los eslabones son rígidos y las juntas son ideales. Se determina las ecuaciones que relacionan las coordenadas del punto $p$ con los valores de las coordenadas de junta $(\theta i, i=1 \ldots 4)$. Se emplean dos ecuaciones de lazo cerrado definidas por los vectores,

$$
\begin{aligned}
& \overrightarrow{O P}=\overrightarrow{O C}+\overrightarrow{C D}+\overrightarrow{D P} \\
& \overrightarrow{O P}=\overrightarrow{O A}+\overrightarrow{A B}+\overrightarrow{B P}
\end{aligned}
$$

Se va a desarrollar el problema de posición para la ecuación (1),

$$
\begin{gathered}
x=l_{1} \cos \left(\theta_{1}\right)+l_{2} \cos \left(\theta_{3}\right)+\frac{d}{2} \\
y=l_{1} \sin \left(\theta_{1}\right)+l_{2} \sin \left(\theta_{3}\right)
\end{gathered}
$$


La variable de interés es $\theta_{l}$ ya que es la coordenada independiente o junta actuada (donde se colocan los motores). La ecuación que la relaciona con $x$ e $y$ se obtiene despejando hacia el lado derecho (o izquierdo) de la ecuación (3) la función trigonométrica que contiene la variable $\theta_{3}$. El mismo procedimiento se realiza sobre la ecuación (4). Luego, ambas ecuaciones se elevan al cuadrado y se suman. Al ser $\sin \left(\theta_{3}\right)^{2}+\sin \left(\theta_{3}\right)^{2}=1$, la ecuación resultante es función de $x, y, \mathrm{y} \theta_{l}$,

$$
\begin{aligned}
& x^{2}+y^{2}+\frac{d^{2}}{4}-d x+l_{1}^{2}+\left(d l_{1}+2 x l_{1}\right) \cos \left(\theta_{1}\right)- \\
& 2 y l_{1} \sin \left(\theta_{1}\right)-l_{2}^{2}=0
\end{aligned}
$$

A partir de la ecuación (5) se puede obtener una expresión para la coordenada de la junta 1. Esto es,

$$
\theta_{1}=\operatorname{atan} 2\left(b_{1}, a_{1}\right) \pm \operatorname{atan} 2\left(\sqrt{a_{1}{ }^{2}+b_{1}{ }^{2}-c_{1}{ }^{2}}, c_{1}\right)
$$

siendo $\quad a_{1}=d l_{1}-2 x l_{1}, \quad b_{1}=-2 y l_{1}, \quad$ y $\quad c_{1}=l_{2}^{2}-$ $\left(x^{2}+y^{2}+\frac{d^{2}}{4}-d x+l_{1}^{2}\right)$

Siguiendo un procedimiento similar para la ecuación (2), se obtiene una ecuación que es función de $x, y, \mathrm{y} \theta_{2}$,

$$
\begin{aligned}
& x^{2}+y^{2}+\frac{d^{2}}{4}-d x+l_{1}{ }^{2}-\left(d l_{1}+2 x l_{1}\right) \cos \left(\theta_{2}\right)- \\
& 2 y l_{1} \sin \left(\theta_{2}\right)-l_{2}{ }^{2}=0
\end{aligned}
$$

A partir de la ecuación (7) se puede obtener una expresión para la coordenada de la junta 2. Esto es,

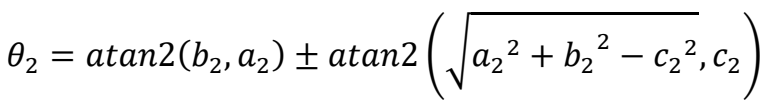

siendo $a_{2}=-d l_{1}-2 x l_{1}, b_{2}=-2 y l_{1}$, y $c_{2}=l_{2}^{2}-$ $\left(x^{2}+y^{2}+\frac{d^{2}}{4}+d x+l_{1}^{2}\right)$

Para determinar los ángulos $\theta_{3}$ y $\theta_{4}$ se realiza un procedimiento similar, pero en este caso de la ecuación (1) se despeja la función trigonométrica relacionada con $\theta_{1}$ y de la ecuación (2) la relacionada con $\theta_{4}$. Luego se elevan al cuadrado y se suman. De las ecuaciones obtenidas se puede llegar a las siguientes ecuaciones,

$$
\theta_{3}=\operatorname{atan} 2\left(b_{3}, a_{3}\right) \pm \operatorname{atan} 2\left(\sqrt{a_{3}{ }^{2}+{b_{3}}^{2}-c_{3}{ }^{2}}, c_{3}\right)
$$

siendo $a_{3}=d l_{2}-2 x l_{2}, b_{3}=-2 y l_{2}, \mathrm{y} c_{3}=l_{1}{ }^{2}-$ $\left(x^{2}+y^{2}+\frac{d^{2}}{4}-d x+l_{2}^{2}\right)$

$$
\theta_{4}=\operatorname{atan} 2\left(b_{4}, a_{4}\right) \pm \operatorname{atan} 2\left(\sqrt{a_{4}{ }^{2}+b_{4}{ }^{2}-c_{4}{ }^{2}}, c_{4}\right)
$$

siendo $a_{4}=-d l_{2}-2 x l_{2}, b_{3}=-2 y l_{2}$, y $c_{3}=l_{1}{ }^{2}-$ $\left(x^{2}+y^{2}+\frac{d^{2}}{4}+d x+l_{2}^{2}\right)$

Es de destacar que la función atan2 se refiere a la tangente del ángulo doble.

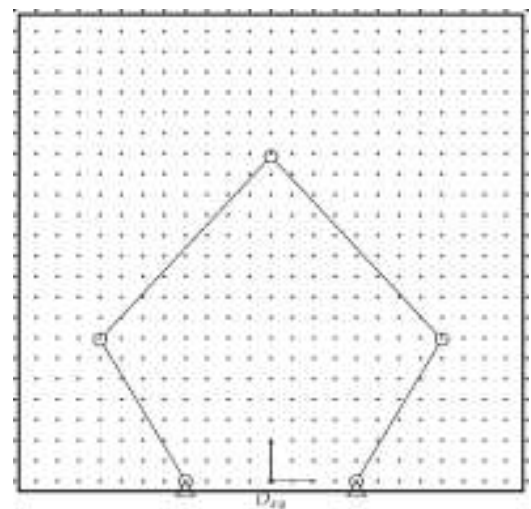

Figura 2. Distribución de puntos uniforme para estimar el valor del espacio de trabajo.

El conjunto definido por las 4 ecuaciones dadas en (5) y (7) define la relación entre las coordenadas actuadas $\theta_{1}$ y $\theta_{2}$ y las coordenadas del punto $p(x, y)$. Si se derivan las ecuaciones respecto al tiempo y se expresan en forma matricial, las velocidades de las juntas actuadas y la velocidad del punto $\mathrm{p}$ se llega a la siguiente expresión,

$$
\left[\begin{array}{l}
\dot{\theta}_{2} \\
\dot{\theta}_{1}
\end{array}\right]=J\left[\begin{array}{l}
\dot{x} \\
y ́
\end{array}\right]
$$

donde, $J=J_{\theta}^{-1} J_{x}$

Así,

$J_{x}=\left[\begin{array}{ll}2 x+d-2 l_{1} \cos \left(\theta_{2}\right) & 2 y-2 l_{1} \sin \left(\theta_{2}\right) \\ 2 x-d-2 l_{1} \cos \left(\theta_{1}\right) & 2 y-2 l_{1} \sin \left(\theta_{1}\right)\end{array}\right]$

Se define como índice de destreza o destreza el número de condición de la matriz Jacobiana $k=\operatorname{cond}(J)$ [5]. Es de resaltar que el índice es local y evalúa la capacidad de desplazarse en ambas direcciones $(x, y)$ en el cual se encuentra el punto $p$, es decir, la destreza del robot. Cuando el $\operatorname{cond}(J)=1$ se tiene la misma habilidad de desplazarse en todas las direcciones. En la medida en que $\operatorname{cond}(J) \rightarrow \infty$, el punto $p$ solo podrá desplazarse en una dirección o quedará imposibilitado de moverse (configuración de singularidad).

La función objetivo requiere determinar el espacio de trabajo y sobre ese espacio evaluar la destreza de cada 
punto perteneciente a dicho espacio. Resulta elevadamente complicado obtener analíticamente el valor del espacio de trabajo, por lo que seguidamente se propone dos procedimientos numéricos para su determinación.

\subsection{Determinación del Espacio de Trabajo}

El espacio de trabajo se determina utilizando la técnica basada en el método de Montecarlo. Para ello, se encierra un área de búsqueda, la cual puede ser definida como un área levemente mayor al tamaño de la mesa de corte requerida. Se selecciona un conjunto de puntos dentro del área, que en este trabajo se realiza mediante dos procedimientos. El primer procedimiento se basa en generar una nube de puntos igualmente espaciada en $x$ e $y$, ver Figura 2. Para determinar el espacio entre la nube de puntos, se hizo un estudio de la precisión en estimar el área en función del número de puntos utilizados. Seguidamente, se evalúa si el punto corresponde a una posición alcanzable por el robot o no, ver Figura (3).

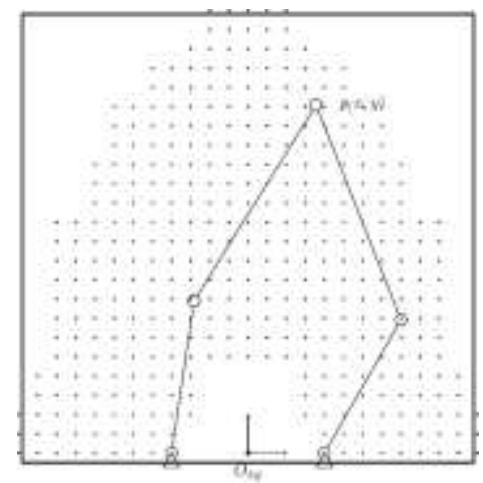

Figura 3. Puntos que son alcanzables por el RP.

El espacio de trabajo (workspace) se estima a partir de la expresión,

$$
A=\frac{\sum_{i=1}^{n} \sum_{j=1}^{m} W_{i j}}{(n m)} A_{t}
$$

donde $A_{t}$ es el área del rectángulo inicial utilizado para estimar el espacio de trabajo, $W_{i j}$ es una matriz que contiene 1 si el punto puede ser alcanzado y 0 si el punto no puede ser alcanzado. Las variables $n$ y $m$ indican el número de puntos discretos utilizados a lo largo de los ejes $x$ e $y$.

Para verificar si el punto es alcanzable $\left(W_{i j}=1\right)$ o no por el robot debe tenerse en cuenta que las ecuaciones (6), (8)-(10) tiene solución factible siempre y cuanto el argumento de la raíz cuadrada sea positivo. Esto es,

donde $k=1 . .4$.

$$
\begin{gathered}
W_{i j}=1 s i \\
a_{k}{ }^{2}+b_{k}{ }^{2}-c_{k}{ }^{2}>0
\end{gathered}
$$

El segundo procedimiento de estimación del espacio de trabajo consiste en una distribución aleatoria de puntos. Se toma como inicio el mismo número de puntos generados por un área discreta de $n \times m$, pero en este caso los puntos se generan aleatoriamente. Para determinar los valores $x$ e $y$ se utiliza la siguiente expresión,

$$
\begin{aligned}
& x=\frac{l_{x}}{2}(2 \text { rand }-1) \\
& y=l_{y}(2 \text { rand }-1)
\end{aligned}
$$

donde $l_{x}$ es el ancho del área de búsqueda y $l_{y}$ la altura.

\subsection{Determinación del Espacio de Trabajo Diestro}

El espacio diestro se determina como aquellos puntos que además de ser alcanzables por el robot debe cumplir la ecuación,

$$
\operatorname{cond}(J)<k
$$

donde $k$ es un número que indica la capacidad del robot en desplazarse con agilidad en todas las direcciones, es decir, la destreza.

\subsection{Descripción del Algoritmo Genético}

El interés de emplear los AG es que permite encontrar el mínimo global. Para una completa descripción del método se puede consultar [18]. Se incluye la Figura 4 a modo de tener una descripción general del algoritmo. El algoritmo inicia con la generación aleatoria de un número de elementos que constituyen posibles soluciones (cromosomas), al conjunto de elementos se les denomina población (PS). La generación se realiza de forma aleatoria generando valores entre 0 y 1 (genes) que forman parte de un carácter de longitud específica. La longitud del carácter (SL) es otro de los parámetros del algoritmo. A mayor longitud se tiene mayor precisión de solución, sin embargo, si es muy elevado el coste computacional hace que la solución requiera de un tiempo computacional elevado e impráctico. Cada cromosoma es decodificado en su valor en el espacio real de la variable (fenotipo) que son empleados para evaluar la función de adaptación (fitness). Dependiendo del valor de la función de adaptación unos cromosomas sobrevivirán hacia la siguiente generación y otros no. A partir de los que sobreviven, se genera una siguiente generación empleando operadores genéticos tales como cruce (crossover), mutación (mutation) copia, o combinación de dichos operadores. Cada operador genético actúa sobre un porcentaje específico de la población por lo que los parámetros porcentaje de cruce (PC) y porcentaje de mutación (PM) deben ser tomados en cuenta para la puesta a punto del AG. El algoritmo se ejecuta iterativamente generación tras generación hasta que algún criterio fijado para terminar el proceso se 
cumpla. Por ejemplo, se puede utilizar como criterio de parada el número máximo de generaciones (iteraciones).

\section{RESULTADOS}

\subsection{Caso Estudio 1}

\subsubsection{Discretizado para determinar el espacio de trabajo.}

Se requiere determinar las longitudes de las barras del robot para que el área alcanzable y diestra sea máxima.

El robot va ser diseñado para una mesa de corte de $1.2 \mathrm{~m}$ de ancho x $1 \mathrm{~m}$ alto. Los intervalos de búsqueda para los longitudes de las barras son $l_{1}=(0.1,0.5), l_{2}=$ $(0.1,0.4)$ y $d=(0.1,0.3)$. Dado que el algoritmo propuesto depende de la cantidad de puntos discretos empleados para estimar el espacio de trabajo, se realiza un estudio de sensibilidad del número de puntos respecto a una solución considerada "exacta". Se toma como solución de referencia o exacta la obtenida con una discretización de paso fino $(\mathrm{h}=0.001 \mathrm{~m})$ rejilla. El equivalente en Monte Carlo aleatorio es de 12221 puntos.

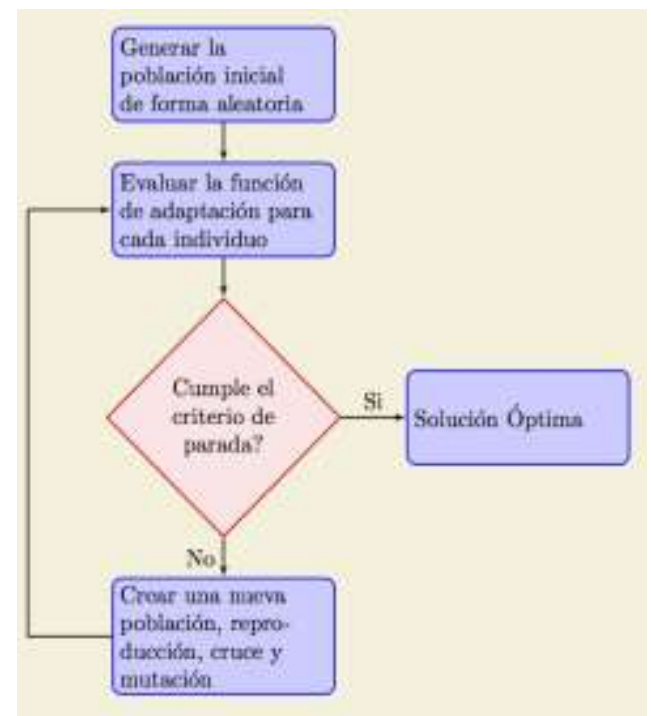

Figura 4. Diagrama esquemático de un AG.

Para un conjunto de 10 parámetros $\left(l, l_{2}, d\right)$ generados de forma aleatoria dentro de los límites de búsqueda, se determinó el valor del área alcanzable y se comparó con la solución de referencia utilizando el error relativo porcentual. A partir del análisis se determinó que con una rejilla de $\mathrm{h}=0.01 \mathrm{~m}$ o 700 puntos se obtiene un error respecto a la solución considerada exacta de media relativa porcentual 0.694 con una desviación estándar de 0.2129. Es de destacar que cada paso o conjunto de puntos aleatorios se ejecutó el algoritmo de determinar el espacio de trabajo para un total.

\subsubsection{Ajuste de los parámetros del AG.}

Se estudió la influencia de los distintos parámetros del AG. La Tabla 1 muestra los parámetros, así como también el conjunto de valores utilizados para ajustar el algoritmo. Se realizó una búsqueda variando un parámetro a la vez. En la tabla, $\mathrm{PC}=$ Porcentaje de Cruce, $\mathrm{PM}=$ Porcentaje de Mutación, $\mathrm{PS}=$ Tamaño de la Población, SL= Longitud del carácter de codificación y Gen= Número de Generaciones.

Tabla 1. Valores utilizados para ajustar el AG Caso 1.

\begin{tabular}{|c|c|c|c|c|c|c|}
\hline PC & 0.6 & 0.65 & 0.70 & $\mathbf{0 . 7 5}$ & 0.85 & 0.90 \\
\hline PM & 0.10 & 0.07 & $\mathbf{0 . 0 5}$ & 0.02 & 0.01 & 0.00 \\
\hline PS & 50 & 100 & 150 & 300 & $\mathbf{4 0 0}$ & 1000 \\
\hline SL & 9 & 12 & $\mathbf{1 5}$ & 18 & 21 & 25 \\
\hline Gen & 100 & 200 & $\mathbf{3 0 0}$ & 400 & 500 & 600 \\
\hline
\end{tabular}

A modo de ejemplo, la Figura 5 muestra los resultados obtenidos para la función de aptitud (fitness) cuando se estudia la variación del PM.

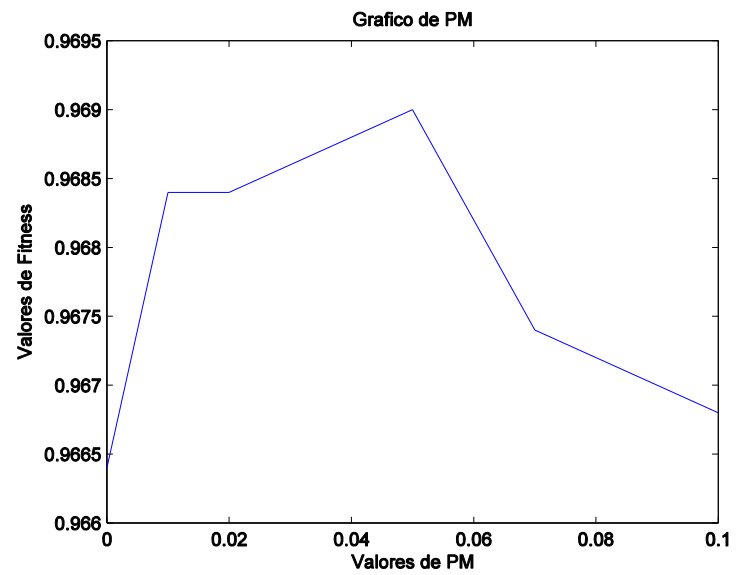

Figura 5. Resultado del valor del fitness para diferentes valores de PM.

\subsubsection{Optimización área alcanzable.}

Luego del estudio exhaustivo se seleccionaron los parámetros resaltados en negrita en la Tabla 1 para la determinación del espacio de trabajo del robot. La optimización se ejecuta 10 veces utilizando cruce de 1 y 2 puntos- Se obtuvo que el valor optimo viene dado por,

$$
(0.5,0.4,0)
$$

cuyo valor de área alcanzable es $0.9715 \mathrm{~m}^{2}$.

La Figura 6 muestra el resultado del área de trabajo obtenida sobre el área de la mesa de corte. 


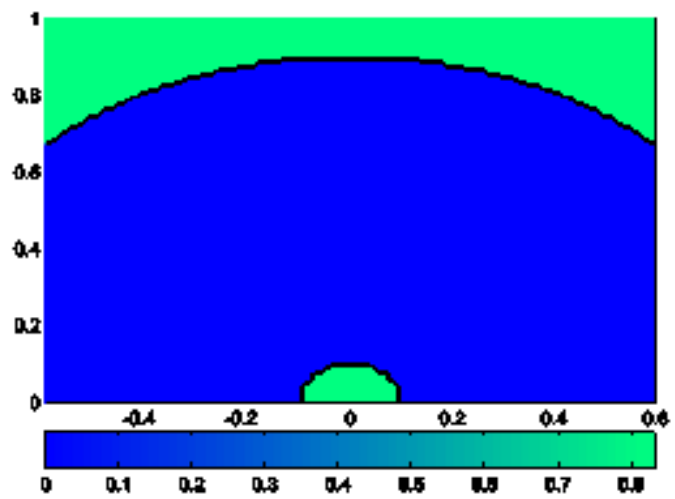

Figura 6. Área alcanzable caso estudio 1.

El resultado obtenido sugiere que si se aumenta los límites de búsqueda, el área alcanzable del robot aumentaría. Es así como se aumenta los límites de búsqueda de los parámetros a: $l_{1}=(0.1,1), l_{2}=(0.1,1)$ y $d=(0.1,0.6)$. En este caso luego de ejecutar la optimización 10 veces, se obtuvo dos posibles soluciones óptimas que abarcan la totalidad del espacio de trabajo requerido. Las soluciones son,

Es de destacar que, si bien las soluciones obtenidas abarcan el espacio requerido por diseño, el robot presenta poca destreza, es decir, es un robot torpe que, aunque puede alcanzar los puntos dentro del espacio de trabajo, estos los alcanza con poca destreza. Es necesario para el diseño del robot no solo considerar el espacio de trabajo, sino que también se debe evaluar la destreza.

\subsection{Caso Estudio 2}

En este caso de estudio se optimiza el espacio diestro. Para ello se emplea como criterio óptimo la ecuación (17). Al igual que en el Caso Estudio 1, se realiza un estudio del número de puntos discretos utilizados, así como como un estudio exhaustivo para ajustar los parámetros del AG. Los valores obtenidos son $\mathrm{PC}=0.70$, $\mathrm{PM}=0.1, \mathrm{SL}=15, \mathrm{PS}=300, \mathrm{Gen}=300$. Se ejecuta el $\mathrm{AG}$ 10 veces tomando en consideración $k=6$. Se obtuvo como resultado,

$$
(0.4,0.4,0.0)
$$

En las optimizaciones se utilizaron cruce de 1 y 2 puntos. Los límites de búsqueda, $l_{1}=(0.1,0.5), l_{2}=(0.1,0.4) \mathrm{y}$ $d=(0.1,0.3)$.Como resultado se tiene un área alcanzable de $0.8468 \mathrm{~m}^{2}$ y destreza de 0.6117. La Figura 7 muestra el espacio de trabajo diestro del robot obtenido.
La franja de colores indica el valor de destreza 1/cond $(J)$. De esta forma un punto de destreza isótropa se da cuando el valor es igual a 1.

Como puede verse en la figura, para los límites de búsqueda y el parámetro de área diestra considerado $(k=6$.), aún hay puntos de poca destreza (franja azul oscuro). Es por lo que se procedió a aumentar el rango de búsqueda de las barras del robot y además si consideró $k=3$ con el objeto de aumentar la destreza.

Los límites de búsqueda para las optimizaciones son $l_{1}=$ $(0.1,1), l_{2}=(0.1,1)$ y $d=(0.1,0.6)$, al igual que todos los casos anteriores, en unas optimizaciones se utiliza cruce de 1 punto y en otras cruce de 2 puntos. La Figura 8 muestra el resultado obtenido. Como puede apreciarse el espacio de trabajo obtenido abarca casi por completo el área de trabajo con un área diestra. La franja roja en la Figura 8 representa la zona donde el robot puede trabajar con mayor destreza.

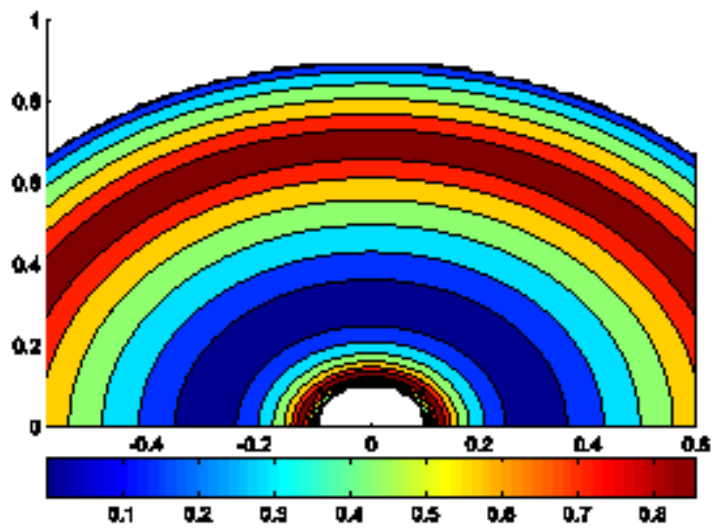

Figura 7. Área Diestra para $(0.4,0.4,0.0)$

\section{DISCUSIÓN}

El estudio de discretización para determinar el espacio de trabajo permite conseguir un compromiso entre tiempo de ejecución del algoritmo y error que se comente con un menor número de puntos discretos. El estudio indica que no es necesario trabajar con una rejilla de paso muy pequeño. Además, entre las dos técnicas utilizadas se recomienda utilizar una rejilla de paso fijo (0.01) y no de generación de puntos aleatorios. De las diferentes ejecuciones del programa elaboradas, el primer método arrojó errores con una media menor que el obtenido con la segunda técnica.

El estudio de los parámetros del AG como son PC, PM, SL, PS y Gen resultó importante ya que permitió obtener resultados consistentes cuando se ejecutan varias optimizaciones. Se recomienda repetir la optimización por AG varias veces ya al ser un método no 
determinístico cada ejecución tiene la probabilidad de llegar a otra solución. Ajustar adecuadamente los parámetros permite soluciones consistentes.

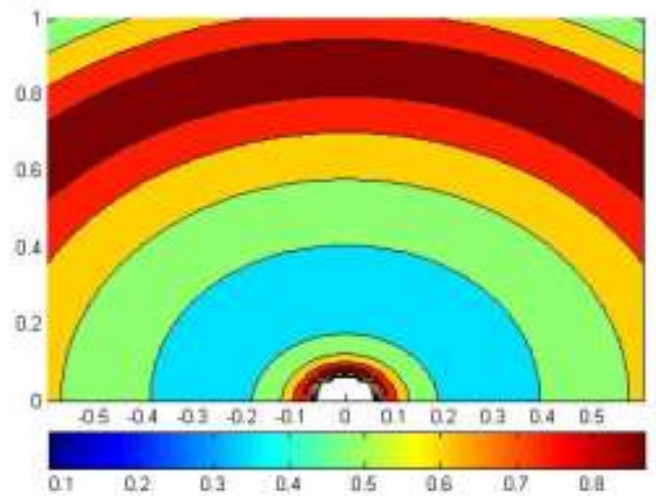

Figura 8. Área Diestra para (0.618,0.675,0.00).

Para el diseño de robot se recomienda que deben emplearse el criterio de destreza, ya que solo el área alcanzable no es suficiente, puede darse el caso que un robot tenga el área alcanzable deseada pero con poca destreza lo cual lo convertiría en un robot torpe.

\section{CONCLUSIONES}

La creciente demanda de los Robot Paralelos por el sector industrial, ha permitido que entes académicos se interesen en el estudio de métodos para resolver problemas actuales en este tipo de robot de complejidad en su topología, así como profundizar en los diferentes temas de estudio como su espacio de trabajo.

En este trabajo se optimizó el espacio de trabajo diestro utilizando Algoritmos Genéticos. Se presentó uno de los problemas de los robots paralelos, que es su poco espacio alcanzable que es pequeño siendo uno de las pocas virtudes de este tipo de robot. En este sentido, se desarrolló un procedimiento numérico para realizar la optimización del área de trabajo del robot considerando también que el espacio de trabajo sea diestro. El aporte principal de este trabajo es que presenta una ruta a seguir sobre cómo abordar el tema de maximizar el espacio alcanzable y de cómo maximizar el espacio diestro perdiendo menos espacio posible, es decir, un espacio apreciable alcanzable y de buena destreza.

Por otra parte, se enseñó un camino de como ajustar los distintos parámetros del Algoritmo Genético para la convergencia del máximo. Se mostró una forma de trabajar en conjunto de como maximizar el espacio de destreza con lo que definimos área de destreza que fue el índice con el que se optimizo.

\section{REFERENCIAS}

[1] F. Pierrot, V. Nabat, O. Company, S. Krut, P. Poignet, "Optimal design of a 4-DOF parallel manipulator: From academia to industry". IEEE Transactions on Robotics, vol. 25, no. 2, pp. 213-224, 2009.

[2] Ortiz-Pimiento, N.R., Gómez-Gómez, S. "Desarrollo de un algoritmo genético para el diseño de sistemas de manufactura celular a partir de una nueva función de aptitud". Revista UIS Ingenierías, vol. 6, no.2, Dic, 2007. pp 71-83.

[3] D.A. Bueno-Sierra, R. Martínez-Ángel, "Planeación de trayectorias para un robot en una celda de manufactura." Revista UIS Ingenierías vol. 1, no 2, pp 31-41, Nov, 2002.

[4] J.P. Merlet, "Workspace-oriented methodology for designing a parallel manipulator". In Robotics and Automation, 1996. Proceedings of IEEE International Conference on, 1996, vol. 4, pp. 3726-3731.

[4] J.P. Merlet, "Jacobian, manipulability, condition number, and accuracy of parallel robots". Journal of Mechanical Design, vol. 128, no. 1, pp. 199-206, 2006.

[5] X.J. Liu, J. Wang, G. Pritschow, "Kinematics, singularity and workspace of planar 5R symmetrical parallel mechanisms". Mechanism and Machine Theory, vol. 41, no. 2, pp. 145-169, 2006.

[6] M.A. Laribi, L. Romdhane, S. Zeghloul, "Analysis and dimensional synthesis of the DELTA robot for a prescribed workspace". Mechanism and Machine Theory, vol. 42, no. 7, pp. 859-870, 2007.

[7] J. Wu, T. Li, X. Liu, L.Wang, "Optimal kinematic design of a 2-dof planar parallel manipulator". Tsinghua Science \& Technology, vol. 12, no. 3, pp. 269-275, 2007.

[8] Y. Lou, G. Liu, Z. Li. "Randomized optimal design of parallel manipulators". IEEE Transactions on Automation Science and Engineering, vol. 5, no. 2, pp. 223-233, 2008.

[9] S.D. Stan, V. Maties, V.R. Balan, "Optimal design of parallel kinematics machines with 2 degrees of freedom". In Parallel Manipulators, Towards New Applications, ITech Education and Publishing, 2008, pp. 295-320.

[10] E. Martínez, C.A. Peña, E. Yime, "Diseño óptimo de un robot paralelo con configuración delta para aplicaciones educativas". Revista Educación en Ingeniería, vol. 5, no. 10, pp. 110-119, 2010. 
[11] O. Altuzarra, B. Sandru, E. Amezua, “Análisis de indicadores cinemáticos para el diseño de manipuladores paralelos". In XVIII Congreso Internacional de Ingeniería Mecánica, Ciudad Real España, 2010.

[12] M. Díaz-Rodríguez, X. Iriarte, V. Mata, J. Ros, “On the experiment design for direct dynamic parameter identification of parallel robots". Advanced Robotics, vol. 23, no 3, p. 329-348. 2009.

[13] M.Z. Huang, "Design of a planar parallel robot for optimal workspace and dexterity". International Journal of Advanced Robotic Systems, vol. 8, no. 4, pp. 176-183, 2011.

[15] C.A, Peña-Cortés, E. Martínez-Oviedo, P.F. Cárdenas-Herrera. "Optimización dimensional de un robot paralelo tipo delta basado en el menor consumo de energía”. Ciencia e Ingeniería Neogranadina, vol. 21, no. 1, pp. 73-88, 2011.

[16] B. Bounab, "Multi-objective optimal design based kineto-elastostatic performance for the delta parallel mechanism". Robotica, vol. 34, no. 02, pp. 258-273, 2016.

[17] A. Barraza Cantillo, J. Rúa Charris, J. Sosa Rodríguez, J. Díaz González, E. Yime Rodríguez, J. Roldán Mckinley, "Modelado dinámico del manipulador serial Mitsubishi Movemaster RV-M1 usando SolidWorks", Revista UIS Ingenierías, vol. 15 (2), pp 4962, Jul - Dic, 2016, doi:10.18273/revuin.v15n22016004.

[18] J.H. Holland. Adaptation in natural and artificial systems. Ann Arbor: The University of Michigan Press, 1975. 